\section{Treatment of scalp wounds in head injured patients requiring transfer to a neurosurgical unit}

EDITOR,-Scalp wounds are a common reason for attendance in the accident and emergency (A\&E) department. Some of these cases will have an associated head injury and require transfer to a neurosurgical unit. We examined the treatment of scalp wounds in this group. In a six month period, 142 patients were admitted to the neurosurgical unit with the diagnosis of head injury. Thirty six of these patients had associated scalp wounds and details of this group are presented.

Twelve patients had adequate treatment of the scalp wounds in the A\&E department. In all of these patients the hair around the wound had been removed, the wound cleaned, and the skin closed. Of the remainder, 14 patients had received no treatment of their wounds despite the presence of the wound being clearly documented in the notes. In 10 patients there had been attempts to treat the wounds that were inadequate because of incomplete cleaning (three cases) or poor closure (seven cases). These 24 patients all required further exposure, cleaning, and suturing of their wounds by the neurosurgical staff. Additional wounds that had not been found before transfer were identified in five cases. In one patient the failure to deal adequately with a scalp wound resulted in a marked blood loss which required resuscitation and blood transfusion.

This study indicates that the treatment of scalp wounds in severely head injured patients is often inadequate. The reasons underlying this are not clear. Perhaps there is a feeling that, if the patient is being transferred for neurosurgical assessment, scalp wounds should be left untreated. We suggest that this is not a good standard of care. Furthermore, clinicians involved in the care of trauma victims should not be reluctant to adequately expose scalp wounds. This is the key to proper treatment.

M O FITZPATRICK

K GOYAL

Department of Neurosurgery, Institute of Neurological Sciences, Southern General Hospital NHS Trust, 1345 Govan Road, Glasgow G51 4TF

\section{UK accident and emergency departments and emergency contraception}

EDITOR,-I read the article on the provision of emergency contraception in the accident and emergency (A\&E) department by Gbolade $e t$ al with interest. ${ }^{1}$ Of course this "emergency contraception" is a misnomer in that it is neither an emergency, as there is a $\mathbf{7 2}$ hour time window, nor is it a contraceptive as it acts by stopping implantation of the "conception".

Most departments are trying to target their resources to $A \& E$ patients. By doing this in Lancaster we have, with the help of the health authority and local general practitioners, kept our new patient numbers static at around 35000 a year over the past 10 years. Extra resources have then helped to keep our waiting times down to an average of 30 minutes. The converse of this is that any A\&E department that attempts to be all things to all people will quickly be overwhelmed by the demand of the public for general health care.
The questionnaire was sent to the person in charge of each A\&E unit (of whom one in three did not respond), yet as the survey demonstrated the consultants were rarely involved in prescribing the initial dose. Perhaps a questionnaire sent to all permanent staff grades in A\&E might better reflect the views of the doctors who would be expected to provide the prescription and advise the women.

The issue of deregulation and having the service available "over the counter" at a chemist is a separate issue, although this in itself would have staff issues of training and reluctance on personal, religious, and moral grounds.

RAY MCGLONE Accident and Emergency Department, Royal Lancaster Infirmary, Ashton Road, Lancaster LA1 4RP (e-mail: Ray.McGlone@LAHT.NWEST.NHS.UK)

1 Gbolade BA, Elstein M, Yates D. UK accident and emergency departments and emergency Accid Emerg Med 1999;16:35-8.

\section{The authors reply}

We appreciate Dr McGlone's interest in our paper but his letter shows some misunderstanding of our study. We disagree with his assertion that the phrase "emergency contraception" is a misnomer. The noun "emergency" means an unexpected occurrence, requiring immediate action; "contraception", also a noun, means the prevention of unwanted pregnancy. For a couple facing the risk of an unplanned and unwanted pregnancy, especially after failure of a barrier method of contraception, it is definitely an emergency situation.

The findings of the survey suggested that the willingness to provide the service appeared to be dependent on a multiplicity of interacting factors including the ethos and leadership of the department. Sending the questionnaire to all permanent staff would not have been useful in that if the leadership was not receptive to the idea in the first place, little could be achieved by the supporting staff.

In noting the opposition to "over the counter" availability of emergency contraception, we were highlighting the incongruity between the apparent reluctance of the accident and emergency departments to provide the service and their reluctance to support measures that would remove the pressure being brought on them to provide the service.

\section{Importance of histology}

EDITOR,-A 47 year old male recently presented to this accident and emergency (A\&E) department complaining of a painful lesion on his right forearm. He related this to a pointed wire penetrating his skin three weeks previously. Examination revealed an apparent collection of pus on the right forearm with surrounding induration and redness. Radiography demonstrated no foreign body. This lesion was incised and drained, an appropriate dressing applied, and oral antibiotics prescribed. The patient was discharged.

Seven weeks later the patient experienced a recurrence of pain in his right forearm. Examination again showed an apparently small collection of pus. Incision and drainage revealed an odourless "pearl white material". Histopathological examination returned a diagnosis of pilomatrixoma with "nuclear atypia and focal necrosis".

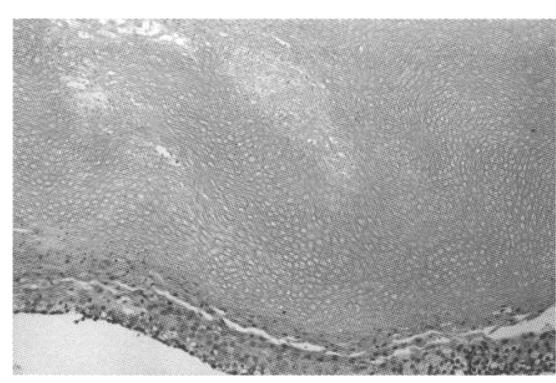

Figure 1 High power view showing basaloid cells in lower part of the field undergoing pilar-type keratinisation, forming a mass of keratinous "ghost" cells. The masses of keratin could be mistaken for a foreign body

(magnification $\times 52$ ).

A pilomatrixoma is a nodular benign tumour arising from the hair matrix. The differential diagnosis includes basal cell carcinoma, keratoacanthoma, and metastasis. It is most often seen in children and young adults, and most cases are located on the head, neck, and upper extremities. Typical features include nests of small basaloid cells, which undergo abrupt keratinisation to form "ghost" and "shadow" cells (fig 1). Extramedullary haematopoiesis, foreign body reaction, calcification, and ossification are seen. An association between pilomatrixoma and trauma has been suggested previously. ${ }^{1}$ Malignant forms of pilomatrixoma are described. ${ }^{2}$ These exhibit cellular atypia, an infiltrating border, mitoses, necrosis, clear cells, and transitions to squamous cells. Local recurrence is seen after inadequate excision. Metastases to distant sites are described. ${ }^{3}$

In view of the atypical features in this case, this man was referred to the dermatology service where he underwent wide local excision of this lesion.

This case demonstrates the importance of obtaining histological examination of all tissue samples when patients undergo minor surgical procedures in an $A \& E$ department. Making a clinical diagnosis without such objective evidence may miss potentially serious conditions, including malignant skin lesions.

P GAFFNEY $S$ WALSH

Accident and Emergency Department, General Infirmary at Leeds, Great George Street, Leeds LS1 3EX

1 Inui S, Kanda $R$, Hata S. Pilomatrixoma with a bullous appearance. $\mathcal{f}$ Dermatol 1997;24:57-9.

2 McCulloch TA, Singh S, Cotton DW. Pilomatrix carcinoma and multiple pilomatrixomas. $B r \mathcal{F}$ Dermatol 1996;134:368-71.

3 Niedermeyer HP, Peris K, Hofler H. Pilomatrix carcinoma with multiple visceral metastases. Report of a case. Cancer 1996;77:1311-4.

\section{Overdose on the internet}

EDITOR,-A 27 year old man attended our accident and emergency department having taken between 60 and $85,60 \mathrm{mg}$ tablets of phenobarbitone and half a bottle of vodka. He complained of dizziness and was noted to be drowsy and have slurred speech. The tablets had been ordered via the internet from a company based in Thailand and were purchased for the sum of $\$ 21$. This was not a suicide attempt but was, he said, an experiment to help him relax. On further questioning he also admitted to taking a wide variety of vitamins and mineral supplements bought from the same company. The patient had a serum phenobarbitone concentration of $71.5 \mathrm{mg} /$ 